\title{
Correlação entre índice de massa corporal e circunferência da cintura em crianças*
}

\author{
Correlation between body mass index and waist circumference in children
}

Correlación entre indice de masa corporal y circunferencia de la cintura en niños

\section{RESUMO}

Objetivo: Estabelecer uma correlação entre a circunferência da cintura e o Índice de Massa Corporal (IMC) na determinação do excesso de peso e distribuição de gordura em crianças de escolas públicas de Fortaleza - CE. Métodos: Trata-se de um estudo transversal, realizado em 12 escolas da rede da pública da cidade de Fortaleza- CE. Participaram 727 crianças de ambos os sexos, com idade variando entre 6 a 11 anos. A coleta de dados ocorreu de março a junho de 2008. Resultados: Os resultados do inquérito mostraram que, de acordo com o IMC,11,4\% das crianças encontravam-se em sobrepeso, 6,6\% eram obesas e 30,9\% apresentaram obesidade central. Conclusão: Nem toda criança obesa apresentava obesidade central, demonstrando que uma só medida antropométrica não é eficaz na determinação do padrão de gordura na infância.

Descritores: Antropometria; Sobrepeso; Criança; Obesidade; Índice de massa corporal

\begin{abstract}
Objective: To establish a correlation between waist circumference and Body Mass Index (BMI) and to determine overweight and fat distribution in children from public schools in Fortaleza - CE. Methods: This is a cross-sectional study conducted in 12 public schools in the city of Fortaleza-CE. The participants were 727 children of both genders, aged 6-11 years. Data collection occurred from March to June 2008. Results: The survey results showed that, according to BMI, $11.4 \%$ of children were overweight, $6.6 \%$ were obese and $30.9 \%$ had central obesity. Conclusion: Not all obese children had central obesity, showing that only one anthropometric measure is not effective in determining the pattern of fat in childhood.
\end{abstract}

Keywords: Anthropometry; Overweight; Child; Obesity; Body mass index

\section{RESUMEN}

Objetivo: Establecer una correlación entre la circunferencia de la cintura y el Índice de Masa Corporal (IMC) en la determinación del exceso de peso y distribución de grasa en niños de escuelas públicas en Fortaleza - CE. Métodos: Se trata de un estudio transversal, realizado en 12 escuelas de la red pública de la ciudad de Fortaleza- CE. Participaron 727 niños de ambos sexos, con edad que varió de 6 a 11 años. La recolección de datos ocurrió de marzo a junio de 2008. Resultados: Los resultados de la encuesta mostraron que, de acuerdo con el IMC, $11,4 \%$ de los niños se encontraban con sobrepeso, 6,6\% eran obesos y 30,9\% presentaron obesidad central. Conclusión: No todos los niños obesos presentaban obesidad central, demostrando que solo una medida antropométrica no es eficaz para determinar el estándar de adiposidad en la infancia.

Descriptores: Antropometría; Sobrepeso; Niño; Obesidad; Índice de massa corporal

\footnotetext{
* Trabalho realizado no Departamento de Enfermagem da Universidade Federal do Ceará. Ceará(CE), Brasil.Trabalho financiado pelo CNPq.

${ }^{1}$ Doutora. Professora do Departamento de Enfermagem da Universidade Federal do Ceará -UFCE - Fortaleza (CE) Brasil.

${ }^{2}$ Pós-graduanda (Mestrado) em Enfermagem pela Universidade Federal do Ceará - UFCE - Fortaleza (CE). Brasil.

${ }^{3}$ Enfermeira da Unidade de AVC do Hospital Geral de Fortaleza - Fortaleza (CE), Brasil.

${ }^{4}$ Mestranda em Enfermagem pela Universidade Federal do Ceará - UFCE- Fortaleza (CE).

${ }^{5}$ Enfermeira do Programa de Saúde da Família de Morada Nova - Morada Nova (CE), Brasil.
} 


\section{INTRODUÇÃO}

A infância é uma fase de intenso e rápido crescimento e desenvolvimento físico, psíquico e social, o que ocasiona um aumento das necessidades nutricionais. Os avanços da vida moderna têm ocasionado mudanças no estilo de vida das famílias brasileiras e de todo o mundo, fazendo com que essas necessidades nutricionais sejam supridas de forma inadequada, por meio do consumo de dietas hipercalóricas, modismos, voracidade alimentar, troca de refeições por lanches rápidos, aleatoriamente, escolhidos ${ }^{(1)}$. Esses fatores unem-se à inatividade física, resultado das mudanças nas brincadeiras infantis que, atualmente, se concentram nos jogos de vídeo e no uso excessivo do computador e televisão.

Os aspectos supracitados corroboram para o aumento da prevalência da obesidade na infância, uma doença crônica caracterizada pelo aumento da gordura corporal e influenciada por fatores genéticos que, aliados aos fatores ambientais, dificultam a manutenção do peso saudável ${ }^{(2)}$. Existem dois tipos de obesidade: a subcutânea que acumula gordura nos quadris e coxas, e a visceral, que é o acúmulo de gordura na região do abdome ${ }^{(3)}$.

No que diz respeito à identificação de casos de obesidade em crianças, uma questão importante vem sendo discutida, ou seja, qual o método mais acurado para classificá-la.

$\mathrm{O}$ índice de massa corporal (IMC) obtido por meio da divisão do peso corporal, em quilo, pela altura em metros quadrados ${ }^{(4)}$, é uma medida antropométrica largamente utilizada na identificação do excesso de peso em crianças, adolescentes e adultos. Todavia, pouco contribui para a determinação do tecido adiposo, já que não distingue a massa gorda da massa muscular, e assim, não representa a distribuição da gordura corporal ${ }^{(5)}$.

Em contrapartida, a circunferência da cintura (CC) definida por meio da medida da menor circunferência entre a crista ilíaca e o rebordo costal é, particularmente, melhor preditora da obesidade visceral, condição que representa alto risco para o desenvolvimento de doenças crônicas não transmissíveis como Diabetes mellitus tipo 2 e doenças cardiovasculares e, dessa forma, vem merecendo destaque nos estudos nacionais e internacionais ${ }^{(6-7)}$.

Além do IMC e da CC, outro método vem sendo utilizado para avaliar a composição corporal total e regional da massa magra, massa gorda e densidade mineral óssea em países como os Estados Unidos da América e Nova Zelândia. Trata-se do Dual-energy X-ray absorptiometry (DEXA), uma técnica de "escaneamento" considerada fidedigna, cujo mecanismo se dá por meio de dois feixes de raios X que atravessam o corpo. No entanto, em se tratando de crianças, embora seja um método de referência, a exposição à radiação e o alto custo para a realidade brasileira devem ser considerados ${ }^{(4,8)}$.

A respeito do conhecimento produzido sobre o uso do IMC ou da CC para avaliar o padrão de gordura corporal, estudiosos do assunto na busca de uma resposta, demonstraram haver um coeficiente de correlação forte entre ambas as medidas, indicando que a circunferência da cintura consegue determinar, satisfatoriamente, crianças com IMC elevado ${ }^{(9-15)}$. Ainda na literatura internacional, destacam-se estudos que concluíram que, dentre as medidas antropométricas, a CC é a que apresenta forte correlação com a distribuição de gordura apontada pelo $\operatorname{DEXA}^{(5,8)}$.

Investigações realizadas no Brasil encontraram valores de correlação importantes entre o IMC e a CC, sugerindo o uso em conjunto para o diagnóstico da obesidade, sobrepeso e obesidade central ${ }^{7,10,16-17)}$.

Inquéritos que relacionam o índice de massa corporal e a circunferência da cintura em crianças e adolescentes ainda são considerados escassos ${ }^{(6,14-15)} \mathrm{e}$, além disso, ao contrário do que ocorre em adultos, não há consenso sobre os critérios antropométricos mais adequados para classificar sobrepeso, obesidade e obesidade central na infância e na adolescência, sendo assim necessário aprofundar os estudos nessa perspectiva ${ }^{(18)}$.

Assim sendo, este estudo tem como objetivos: estabelecer correlação entre o IMC e CC; caracterizar a amostra, de acordo com a idade, o sexo e a série e conhecer a prevalência do sobrepeso, obesidade e obesidade central em crianças de escolas públicas de Fortaleza-CE.

\section{MÉTODOS}

Estudo transversal realizado em Fortaleza, no período de janeiro a dezembro de 2008, em 12 escolas públicas da rede municipal. Como a prefeitura da cidade é dividida em seis regionais, para o presente inquérito, foram selecionadas duas escolas de cada regional, situadas em bairros distintos, no que diz respeito à infraestrutura e serviços, com o objetivo de obter dados representativos da cidade em estudo.

Como critérios de inclusão, foram estabelecidos: ter entre 6 e11 anos de idade; estar frequentando a escola no período da coleta de dados; concordar em participar do estudo e apresentar o Termo de Consentimento Livre e Esclarecido assinado pelos pais e responsáveis. A faixa etária referida foi assim delimitada porque, aos 6 anos, a criança da escola pública inicia seus estudos e ainda porque, em pesquisa semelhante realizada na cidade de Fortaleza, se investigou a faixa etária entre 12 e $17 \operatorname{anos}^{(19)}$.

Foram excluídas as crianças com os diagnósticos de Diabetes mellitus tipo 1 ou tipo 2, visto que os dados do presente inquérito foram extraídos de uma investigação realizada com a finalidade de identificar fatores de risco para o desenvolvimento de Diabetes mellitus tipo $2^{(20)}$.

O número total de estudantes da rede pública matriculados na faixa etária de interesse para a pesquisa 
era de 125.526, distribuídos nas seis regionais, conforme os dados da Tabela 1. O tamanho da amostra foi calculado, utilizando-se uma fórmula para população infinita.

$$
\mathrm{N}=\underline{\mathrm{t}}_{\underline{5 \%}}^{2} \frac{\mathrm{XPXQ}}{\mathrm{e}^{2}}
$$

Onde $\mathrm{t}=$ valor da distribuição de Student $\left(\mathrm{t}_{5 \%}=1,96\right)$; $\mathrm{P}=50 \% ; \mathrm{Q}=100 \%-\mathrm{P}=50 \%$; e o erro amostral absoluto $=4 \%$. Consideraram-se os valores supracitados para $\mathrm{P}$ e Q, haja vista que proporciona um tamanho da amostra, além do nível de significância $(a=0,05)$ e o erro amostral absoluto de 4\%.

Assim, o número de participantes determinados foi equivalente a 600 crianças. Em virtude das prováveis perdas, acrescentou-se uma margem de $20 \%$ a esse tamanho amostral e, por isso, o " $n$ " foi de 720 crianças, porém sete crianças expressaram o desejo espontâneo de participar da pesquisa e uma vez atendendo aos critérios de inclusão fizeram parte do estudo, totalizando uma amostra de 727 indivíduos ${ }^{(21)}$.

A partir da amostra total (727 alunos), estabeleceu-se a amostra por região, conforme exposto nos dados da Tabela 1. Essa, por sua vez, foi dividida por dois, o que representa o número de alunos estudados em cada escola da região.

Tabela 1 - Distribuição da amostra por região. FortalezaCE, 2007

\begin{tabular}{|c|c|c|c|c|}
\hline \multirow[t]{2}{*}{ Regiões } & \multirow{2}{*}{$\begin{array}{c}\begin{array}{l}\text { N.o de alunos } \\
\text { matriculados }\end{array} \\
\text { n. }^{\circ} \\
\end{array}$} & \multirow{2}{*}{$\begin{array}{c}\begin{array}{c}\text { Amostra } \\
\text { por região }\end{array} \\
\text { n. }^{\circ}\end{array}$} & \multicolumn{2}{|c|}{$\begin{array}{c}\text { Amostra por } \\
\text { escola }\end{array}$} \\
\hline & & & n. ${ }^{\circ}$ & Escolas \\
\hline \multirow[t]{2}{*}{ I } & 20.295 & 108 & 54 & $\mathrm{~A}$ \\
\hline & & & 54 & B \\
\hline \multirow[t]{2}{*}{ II } & 10.443 & 60 & 30 & $\mathrm{C}$ \\
\hline & & & 30 & $\mathrm{D}$ \\
\hline \multirow[t]{2}{*}{ III } & 16.071 & 103 & 50 & $\mathrm{E}$ \\
\hline & & & 53 & $\mathrm{~F}$ \\
\hline \multirow[t]{2}{*}{ IV } & 8.740 & 52 & 26 & G \\
\hline & & & 26 & $\mathrm{H}$ \\
\hline \multirow[t]{2}{*}{ V } & 35.180 & 204 & 95 & $\mathrm{I}$ \\
\hline & & & 109 & $\mathrm{~J}$ \\
\hline \multirow[t]{2}{*}{ VI } & 34.797 & 200 & 105 & J \\
\hline & & & 95 & $\mathrm{~K}$ \\
\hline Total & 125.526 & 727 & 727 & 12 \\
\hline
\end{tabular}

Fonte: Secretaria Municipal de Educação de Fortaleza - CE

Os dados foram coletados por bolsistas PIBIC/ $\mathrm{CNPq}, \mathrm{IC} / \mathrm{CNPq}$, mestrandos e doutorandos membros do projeto de pesquisa "Ações integradas na prevenção e controle de Diabetes mellitus tipo 2", financiado pelo CNPq, no qual o presente estudo está inserido. Os membros envolvidos receberam rigoroso treinamento que incluiu as técnicas utilizadas para a verificação do peso, da altura e da CC e, ainda, foram capacitados para que dominassem a técnica da entrevista.

A coleta de dados ocorreu no período de março a junho de 2008, por meio de um formulário com tópicos estruturados, nos quais foram registrados: nome, idade, sexo, série, peso, altura, índice de massa corporal (IMC) e circunferência da cintura (CC).

O peso foi obtido em uma única verificação, utilizando-se de uma balança portátil digital da marca PLENA com capacidade de $150 \mathrm{~kg}$ e uma precisão de 0,1 kg. Na ocasião, os indivíduos estavam descalços, com roupas leves e em posição ortostática, conforme orientação, subiram, cuidadosamente, e posicionaram-se no centro da balança.

A altura foi avaliada por meio de uma fita métrica com precisão de $0,5 \mathrm{~cm}$, fixada em parede lisa, com os alunos descalços, de costas para a parede, pés unidos e paralelos, em posição ereta e olhando para a frente. Com a finalidade de assegurar a exatidão da medida da altura na fita métrica, utilizou-se uma régua que foi posta sobre a cabeça dos participantes ${ }^{(2)}$.

Em seguida, foi calculado o IMC, cujos valores permitiram classificar as crianças em sobrepeso e obesidade, considerando os critérios preconizados por estudiosos que afirmam que, tal classificação, em se tratando de crianças e adolescentes varia de acordo com a idade, o sexo e a distribuição do percentil. No caso de uma criança do sexo feminino com idade de seis anos, o valor do IMC correspondente ao sobrepeso seria de $17,55 \mathrm{~kg} / \mathrm{m}^{2}$, estando, portanto, situada no percentil 85 . Por outro lado, o valor correspondente à obesidade seria o IMC de $19,78 \mathrm{~kg} / \mathrm{m}^{2}$, estando inserida no percentil $95^{(22)}$. Vale ressaltar que os referidos valores são também aceitos e recomendados pela International Obesity Task Force ${ }^{(23)}$.

A circunferência da cintura foi mensurada com a criança em posição ortostática, colocando-se sobre a pele uma fita inelástica graduada em milímetros, no espaço correspondente à menor circunferência entre a crista ilíaca e o rebordo costal, no momento da mínima expiração. Foram consideradas com obesidade central, as crianças cujos valores da CC, de acordo com o sexo e a idade, eram iguais ou maiores que os do percentil $80^{(())}$. No caso das características da criança acima referida, o valor da CC correspondente à obesidade central seria de $59,2 \mathrm{~cm}$, estando distribuída no percentil 80.

Os resultados foram analisados com base na literatura específica e apresentados em tabelas e figuras. Foram calculados a média e o desvio padrão, bem como as medidas epidemiológicas, para fazer uma apreciação descritiva dos dados. Para as análises de associação entre variáveis, foram utilizados os testes não paramétricos de Qui-Quadrado e de Fisher; e para testar as médias de variáveis foram aplicados os testes de t de Student e o teste F de Sdenecor. Em todos os testes estatísticos, fixouse o nível de significância de 5\%.

O projeto foi aprovado pelo Comitê de Ética em Pesquisa em Seres Humanos da Universidade Federal do 
Ceará, sob o protocolo nº 18/08.

\section{RESULTADOS}

Foram avaliadas 727 crianças: 395 do sexo feminino $(54,3 \%)$ e 332 do sexo masculino $(45,7 \%)$. As idades prevalentes foram 8 anos $(21,3 \%)$ e 10 anos (21\%), sendo a média de idade de 8,47 anos e 8,67 anos para meninas e meninos, respectivamente. Quanto à série, $26 \%$ das crianças cursavam o terceiro ano do ensino fundamental. Os valores médios do IMC e CC para as crianças em obesidade central foram de $20,65 \mathrm{~kg} \backslash \mathrm{m}^{2}$ e de $73,92 \mathrm{~cm}$, e para as crianças com sobrepeso, foram de $23,4 \mathrm{~kg} \backslash \mathrm{m}^{2}$ e de $71,8 \mathrm{~cm}$ e para as obesas, de $24,1 \mathrm{~kg} \backslash \mathrm{m}^{2}$ e de $79,3 \mathrm{~cm}$, respectivamente.

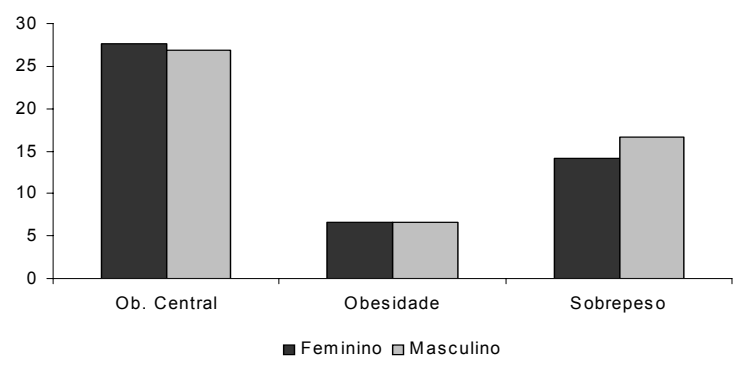

Figura 1 - Prevalência de obesidade central, obesidade e sobrepeso em crianças de 6 - 11 anos, segundo sexo. Fortaleza-CE, 2008.

A avaliação da circunferência abdominal, segundo os valores de referência para sexo e idade ${ }^{(6)}$, evidenciou que a maioria das crianças $(72,8 \%)$ apresentou a CC normal e que em $27,2 \%$ da amostra a obesidade central esteve presente. Quanto ao sexo, a prevalência de obesidade central foi de $27,6 \%$ e $26,8 \%$ entre o sexo feminino e o masculino, respectivamente, conforme a Figura 1.

A análise dos valores do IMC, de acordo com os percentis utilizados como referência para a idade e sexo ${ }^{(21)}$, revelou que a prevalência de crianças obesas para ambos os sexos e para o total de alunos foi de $6,6 \%$, já para o sobrepeso, a prevalência foi de $14,2 \%$ para o sexo feminino e $16,6 \%$ para o sexo masculino, e a prevalência total foi de $15,30 \%$ (Figura 1).

A idade em que a prevalência para sobrepeso, obesidade e obesidade central esteve mais elevada foi aos 9 anos, com $19 \%$, 9,5\% e 35,9\%, respectivamente, seguida dos 6 anos de idade, quando as prevalências foram 17,4\%, 9,3\% e 27,9\%, respectivamente (Figura 2).

É importante salientar que a prevalência total de obesidade central foi maior para ambos os sexos, em comparação com a obesidade e o sobrepeso, sugerindo que mesmo crianças consideradas normais pelo IMC apresentam elevada adiposidade central, o que é preocupante diante dos riscos para doenças crônicas não transmissíveis.

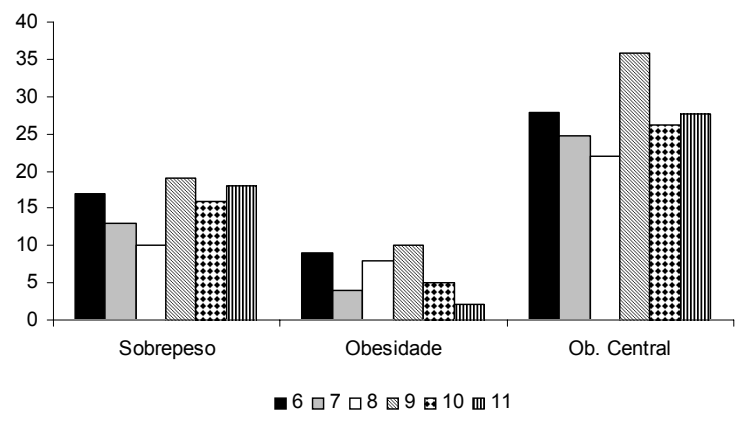

Figura 2 - Prevalência de sobrepeso, obesidade e obesidade central, segundo idade das crianças. FortalezaCE, 2008.

Os dados da Tabela 2 evidenciam que 9,3\% das crianças classificadas como normais pelo IMC, 89,2 \% das crianças como sobrepeso pelo IMC e $95,8 \%$ das crianças obesas também pelo IMC, têm obesidade central quando classificadas pela CC.

Tabela 2 - Escolares, segundo classificação do IMC e da Circunferência da Cintura (CC). Fortaleza-CE, 2008

\begin{tabular}{lcc}
\hline \multirow{2}{*}{ Classificação do IMC } & \multicolumn{2}{c}{ Classificação da CC } \\
\cline { 2 - 3 } & Normal & Obesa \\
\hline Normal & & \\
N & 515 & 53 \\
$\%$ & 90,7 & 9,3 \\
Obeso & & \\
N & 2 & 46 \\
$\%$ & 4,2 & 95,8 \\
Sobrepeso & & \\
N & 12 & 99 \\
$\%$ & 10,8 & 89,2 \\
\hline
\end{tabular}

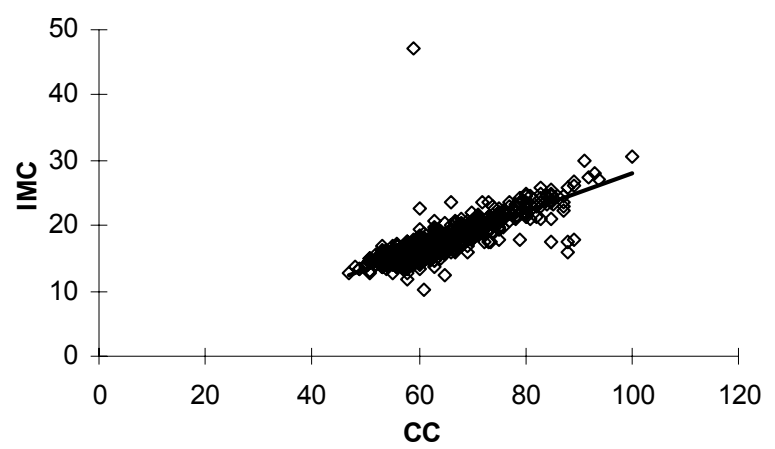

$\diamond \quad$ IMC Previsto(a) IMC L Linear (Previsto(a) IMC)

Figura 3 - Dispersão da circunferência da cintura pelo índice de Massa Corporal com reta ajustada dos escolares de 6-11 anos. Fortaleza-CE, 2008.

O teste estatístico do Qui-quadrado verificou que há indicações da existência de associação entre a classificação 
das crianças quanto ao IMC e a classificação quanto a CC, com $\mathrm{p}<0,001$ e coeficiente de correlação entre as duas classificações de $r=0,816$, com valor $\mathrm{p}<0,001$, sendo considerado forte.

A análise da regressão linear do IMC em função da CC demonstrada, diz que a circunferência da cintura tem uma previsão do IMC de $66,6 \%$ de segurança para um $\mathrm{p}<0,001$, ou seja, $66 \%$ das crianças com CC alterada também apresentavam o IMC alterado (Figura 3).

\section{DISCUSSÃO}

O IMC expressa alterações que podem ocorrer na distribuição de gordura, porém não verifica o padrão de gordura corporal. Assim, correlacionar essa medida com outras medidas antropométricas se faz necessário, tendo em vista que o tipo de depósito de distribuição de gordura está relacionado ao prognóstico de saúde ${ }^{(10)}$. No entanto, a CC é a medida que representa melhor a distribuição da gordura visceral e essa, por sua vez, tem mais relação com as alterações metabólicas do que a gordura subcutânea, indicando o risco da criança ou do adolescente de desenvolver doença cardiovascular na vida futura ${ }^{(17)}$. Além disso, a relação dessa medida antropométrica com a dislipidemia, com a hipertensão arterial e com a síndrome metabólica está evidenciada na literatura e, dessa forma, deve subsidiar a prática profissional ${ }^{(11,24-25)}$.

$O$ estudo ora desenvolvido mostrou uma forte correlação entre o IMC e a CC $(r=0.816$ e p<0,001) a exemplo do que foi encontrado em pesquisas nacionais e internacionais sobre o assunto em pauta ${ }^{(7-8,10-14,16-17)}$. Vale ressaltar que não foram encontrados estudos que tenham mostrado correlação fraca ou nenhuma correlação entre as medidas antropométricas focalizadas nessa pesquisa.

Diante desses resultados, o uso em conjunto do IMC e da CC para o diagnóstico de obesidade e tipo de adiposidade pode-se configurar, como uma evidência a ser utilizada na prática clínica ${ }^{(7,10)}$.

Todavia, a literatura confirma que, para um mesmo valor de IMC, pode haver indivíduos com mais ou menos risco cardiovascular e metabólico, dependendo da quantidade de gordura intraabdominal, o que também é válido para as crianças ${ }^{(12)}$. Os resultados de um inquérito realizado na Argentina reforçam essa assertiva, uma vez que apenas $28,6 \%$ e $87,5 \%$ das crianças com sobrepeso e obesidade, respectivamente, encontravam-se também com obesidade central ${ }^{(11)}$. Isso quer dizer que nem todos os obesos e os com sobrepeso que participaram da pesquisa, tinham obesidade central, resultado considerado semelhante ao revelado no presente estudo.

É importante ressaltar que ainda foram encontrados casos de crianças com o IMC normal, porém com obesidade central (9,3\%). A este respeito, não se encontrou na literatura pertinente nenhuma investigação que permitisse a discussão aprofundada desse achado.

Com relação à prevalência de sobrepeso e obesidade, os resultados do estudo em pauta podem ser considerados aproximados ao de outro inquérito realizado no Nordeste, verificando-se ainda que em ambos os estudos o percentual de sobrepeso foi superior ao de obesidade ${ }^{(26)}$. Em contrapartida no Sul e Sudeste, os casos de obesidade foram superiores aos de sobrepeso, isto é, $15,4 \%$ e $13,5 \%$ versus $8,25 \%$ e $6,0 \%{ }^{(8,26)}$. No tocante à obesidade visceral, encontrou-se que $27,7 \%$ das crianças apresentaram este quadro todavia, investigações realizadas em São Paulo e Espanha revelaram valores de 53\%, $44,3 \%$ e $33 \%$, respectivamente ${ }^{(12,17,28)}$.

Acredita-se que as melhores condições socioecônomicas sejam responsáveis tanto pelos altos índices de obesidade em comparação com as cidades do Nordeste como pelos maiores percentuais em relação ao sobrepeso. Outro aspecto que pode justificar as diferenças nos resultados dos estudos, é a existência, na literatura, de controvérsias a respeito dos percentis que classificam a obesidade, o sobrepeso e a obesidade central ${ }^{(14,22,29-30)}$.

Acrescenta-se às limitações do estudo, a inexistência de pontos de corte padronizados para a classificação de crianças em sobrepeso, obesidade e obesidade central. O erro técnico de verificação das medidas antropométricas para a classificação de crianças em sobrepeso, obesidade e obesidade central não ficou estabelecido. No entanto, o fato de que cada componente tinha sua atribuição específica, contribuiu para minimizar essas limitações.

\section{CONCLUSÕES}

O estudo reiterou que existe correlação forte entre o IMC e a CC e que nem toda criança obesa ou com sobrepeso apresenta obesidade central, como também nem toda criança que tem obesidade central, tem sobrepeso ou obesidade subcutânea, demonstrando que o uso isolado da CC ou do IMC é ineficaz na determinação do padrão de gordura na infância.

Portanto, na prática da enfermagem, sugere-se o uso de ambas as medidas antropométricas estudadas para a determinação da distribuição da gordura em crianças e como meio de triagem para a hipertensão arterial, a resistência insulínica e as dislipidemias em crianças e adolescentes, seja nas unidades básicas de saúde, nas escolas e nos serviços especializados.

Os resultados obtidos poderão alertar as enfermeiras no sentido de tornar rotineira a verificação do IMC e da $\mathrm{CC}$ em suas consultas, visto que se tratam de procedimentos de baixo custo e exequíveis, sobretudo, na atenção básica. 


\section{REFERÊNCIAS}

1. Wong DL. Fundamentos de enfermagem pediátrica. $7^{\mathrm{a}}$. ed. Rio de Janeiro: Elsevier; 2006.

2. Damasceno MMC, Lopes MVO, Oliveira DD, Nogueira NP, Siqueira IA, Macêdo SF. Sobrepeso em adolescentes de escolas públicas: desempenho de três critérios diagnóticos. Acta Paulista de Enfermagem. 2009;22(2):198-204.

3. Cedra MC. Tipos de obesidade. Artigos de Obesidade [Internet] 2008 [citado em 2009 Mar 10]: [cerca de 3p.]. Disponível em : http://www.anutricionista.com/tipos-deobesidade.html

4. Sant'Anna MSL, Priore SE, Franceschini SCC. Métodos de avaliação da composição corporal em crianças [revisão]. Rev Paul Pediatr. 2009;27(3):315-21.

5. Zambon MP, Zanolli ML, Marmo DB, Mgna LA, Guimarey LM. Correlação entre o índice de massa corporal e a prega cutânea tricipital em crianças da cidade de Paulínia, São Paulo, SP. Rev Assoc Med Bras (1992). 2003;49(2):137-40.

6. Taylor RW, Jones IE, Williams SM, Goulding A. Evaluation of waist circumference, waist-to-hip ratio, and the conicity index as screening tools for high trunk fat mass, as measured by dual-energy X-ray absorptiometry, in children aged 3-19 y. Am J Clin Nutr. 2000;72(2):490-5.

7. Soar C, Vasconcelos FAG, Assis MAA. A relação cintura quadril e o perímetro da cintura associados ao índice de massa corporal em estudo com escolares. Cad Saúde Pública = Rep Public Health. 2004;20(6):1609-16.

8. Daniels SR, Khoury PR, Morrison JA. Utility of different measures of body fat distribution in children and adolescents. Am J Epidemiol. 2000;152(12):1179-84.

9. Li C, Ford ES, Mokdad AH, Cook S. Recent trends in waist circumference and waist-height ratio among US children and adolescents. Pediatrics. 2006;118(5):e1390-8.

10. Ricardo GD, Caldera GV, Corso ACT. Prevalência de sobrepeso e obesidade e indicadores de adiposidade central em escolares de Santa Catarina, Brasil. Rev Bras Epidemiol. 2009;12(3):424-35.

11. Hirschler V, Defino AM, Clemente G, Arana C, Calcagno ML, Pettinicchio H, Jadzinsky M. Es la circunferencia de cintura un componente del síndrome metabólico en la infância? Arch Argent Pediatr. 2005;103(1):7-13.

12. Méndez MJB, Muíño MJ, Celemin IC, Segade XB, Fernández MF. Cad Aten Primaria. 2007;13(4):242-7.

13. Pajuelo Ramírez J, Canchari E, Léguía D. La circunferencia de la cintura en niños con sobrepeso y obesidad. An Fac Med (Peru). 2004;65(3):167-71.

14. Fredman DS, Serdula MK, Srinivasan SR, Berenson GS. Relation of circumferences and skinfold thicknesses to lipid and insulin concentrations in children and adolescents: the Bogalusa Heart Study. Am J Clin Nutr. 1999;69(2):308-17.

15. Savva SC, Tornaritis M, Savva ME, Kourides Y, Panagi A, Silikiotou N, et al. Waist circumference and waist-to-height ratio are better predictors of cardiovascular disease risk factors in children than body mass index. Int J Obes Relat Metab Disord. 2000;24(11):1453-8.

16. Giugiliano R, Melo ALP. Diagnóstico de sobrepeso e obesidade em escolares: utilização do índice de massa corporal segundo padrão internacional. J Pediatr (Rio J). 2004;80(2):129-34.
17. Pierine DT, Carrascosa APM, Fornazari AC, Watanabe MT, Catalani MCT, Fukuju MM, et al. Composição corporal, atividade física e consumo alimentar de alunos do ensino fundamental e médio. Motriz. 2006;12(2):113-24.

18. Araújo TL, Lopes MVO, Oliveira ARS, Chaves DBR, Costa AGS, Alves FEC, et al. Fatores de risco para hipertensão arterial em escolares: estudo de caso-controle. Rev Enferm UERJ. 2008;16(2):149-55.

19. Silva ARV. Prevenção de Diabetes Mellitus tipo $2 \mathrm{em}$ adolescentes de risco: avaliação de duas estratégias de educação [tese]. Ceará: Universidade Federal do Ceará. Departamento de Enfermagem; 2009.

20. Macêdo SF. Fatores de risco para Diabetes Mellitus tipo 2 em crianças de escolas públicas de Fortaleza-CE [tese]. Ceará: Universidade Federal do Ceará- Departamento de Enfermagem; 2009.

21. Yanovski SZ, Yanovski JA. Obesity. N Engl J Med. 2002;346(8):591-602. Review.

22. Cole TJ, Bellizzi MC, Flegal KM, Dietz WH. Establishing a standard definition for child overweight and obesity worldwide: international survey. BMJ. 2000;320(7244): 12403. Comment in: BMJ. 2000;321(7269):1158-9. BMJ. 2000;321(7269):1158. BMJ. 2000;321(7269):1159. BMJ. 2000;321(7269):1159. BMJ. 2001;323(7319):999.

23. Soares NT. Um novo referencial antropométrico de crescimento: significados e implicações. Rev Nutr. 2003;16(1):93-104.

24. Sarni RS, Souza FIS, Schoeps DO, Catherino P, Oliveira MCCP, Pessotti CFX, et al. Relação da cintura abdominal com a condição nutricional, perfil lipídico e pressão arterial em pré-escolares de baixo estrato socioeconômico. Arq Bras Cardiol. 2006;87(2):153-8.

25. Lunardi CC, Petroski EL. Índice de massa corporal, circunferência da cintura e dobra cutânea triciptal na predição de alterações lipídicas em crianças com 11 anos de idade. Arq Bras Endocrinol Metab. 2008;52(6):1009-14.

26. Oliveira AMA, Cerqueira EMM, Souza JS, Oliveira AC. Sobrepeso e obesidade infantil: influência de fatores biológicos e ambientais em Feira de Santana, BA. Arq Bras Endocrinol Metab. 2003;47(2):144-50.

27. Soleto YOM, Colugnati FAB, Taddei JAAC. Prevalência de sobrepeso e obesidade entre escolares da rede pública segundo três critérios de diagnóstico antropométrico. Cad Saúde Pública = Rep Public Health. 2004;20(1):233-40.

28. Costa RF. Distribuição de valores de medidas antropométricas e avaliação do estado nutricional de escolares de 7 a 10 anos de idade, da cidade de Santos - SP [tese]. São Paulo: Universidade Federal de São Paulo. Escola Paulista de Medicina; 2006.

29. Must A, Dallal GE, Dietz WH. Reference data for obesity: 85th and 95th percentiles of body mass index (wt/ht2) and triceps skinfold thickness. Am J Clin Nutr. 1991;53(4):83946. Erratum in: Am J Clin Nutr 1991;54(5):773. Comment in: Am J Clin Nutr. 1991;54(5):953-6.

30. World Health Organization. Obesity: preventing and managing the global epidemic: report of a WHO consultation on obesity. Geneva: World Health Organization; 1998. 